\title{
Mueller Coherency matrix method for contrast image in tissue polarimetry
}

\author{
J. L. Arce-Diego*, F. Fanjul-Vélez*, D. Samperio-García, D. Pereda-Cubián \\ Applied Optical Techniques Group, TEISA Department, University of Cantabria, Av. de los Castros \\ S/N, 39005 Santander, Cantabria (Spain)
}

\begin{abstract}
In this work, we propose the use of the Mueller Coherency matrix of biological tissues in order to increase the information from tissue images and so their contrast. This method involves different Mueller Coherency matrix based parameters, like the eigenvalues analysis, the entropy factor calculation, polarization components crosstalks, linear and circular polarization degrees, hermiticity or the Quaternions analysis in case depolarisation properties of tissue are sufficiently low. All these parameters make information appear clearer and so increase image contrast, so pathologies like cancer could be detected in a sooner stage of development. The election will depend on the concrete pathological process under study. This Mueller Coherency matrix method can be applied to a single tissue point, or it can be combined with a tomographic technique, so as to obtain a $3 \mathrm{D}$ representation of polarization contrast parameters in pathological tissues. The application of this analysis to concrete diseases can lead to tissue burn depth estimation or cancer early detection.
\end{abstract}

Keywords: tissue polarimetry, Mueller matrix, Mueller Coherency matrix, entropy, backscattering polarimeter

\section{INTRODUCTION}

Optical techniques in characterization of biological tissues present advantages like being harmless, non-invasive, without contact and with a very good resolution [1]. Optical characterization of tissues is usually related with intensity measurements, what allows the achievement of partial optical information from tissues. The fact that most tissues have intrinsic anisotropy and/or structural anisotropy, makes that polarization parameters can add important information to the images acquired, in such a way that hidden compounds or structures, significant from the point of view of diagnosis, may appear. Furthermore, scattering from tissues, that can also be anisotropic, changes the degree of polarization of light and this is of course reflected in polarization parameters [2]. For instance, blood or adipose tissue present no anisotropy but scattering due to the particles involved in their composition. On the other hand, collagen fibers like tendons show anisotropy as a consequence of their structural orientation. Polarimetry is an optical technique focused on the measurement of polarization properties of samples including the properties of depolarizing optical media. Polarimetric techniques are specially appropriate for biological tissues, due to the fact that their properties show dependence with the polarization of light, and they usually exhibit a depolarising behaviour. Methods of analysis that do not take into account tissue depolarisation, like Jones matrix, produce limited results [3]. The extension of these characterization techniques to Mueller matrix measurement adds data to the image obtained, but further information can be extracted [4].

In this work, a complex Mueller matrix analysis of biological tissue images is proposed, by means of significant and clearer parameters. Section 2 describes the main concepts of the Mueller polarimetry and the Mueller Coherency matrix method, the entropy factor, the horizontal-to-vertical cross-talk $(H V C)$ as well as the right-to-left cross-talk $(R V C)$ and the different degrees of polarization, linear and circular, are included. Lu-Chipman decomposition is also described as another approach to the study and decomposition of Mueller matrix. This method allows the extraction of information related with diattenuation, rotation and depolarisation by means of specifically obtained new matrices. In section 3 the results obtained applying the potentiality of the Mueller polarimetry previously described to the concrete Mueller matrices of two different suspensions that were measured in backscattering are presented. Their parameters are calculated and a discussion is included in section 4. As a final step, conclusions of the whole work are provided.

"jlarce@teisa.unican.es; ffanjul@teisa.unican.es: phone +34942201545; fax +34942201873; www.teisa.unican.es/toa

Optical Coherence Tomography and Coherence Techniques III, edited by Peter E. Andersen, Zhongping Chen, Proc. of SPIE-OSA Biomedical Optics, SPIE Vol. 6627, 66271T, (c) 2007 SPIE-OSA · 1605-7422/07/\$18 


\section{MUELLER POLARIMETRY ANALYSIS}

Biological tissues present in general a very high degree of heterogeneity and then they depolarise the optical radiation when irradiated [1]. Due to this behaviour the Mueller matrices are the most useful tool in order to study polarization dependent interaction between light and tissues. The Mueller matrix has 16 elements, and they include all the polarization dependent properties of the tissue [5].

The aim of this section is the theoretical description of the Mueller matrix polarimetry, focused both in the Mueller Coherency matrix description and in the Lu-Chipman decomposition.

\subsection{Mueller Coherency matrix method}

The Dirac matrices $\boldsymbol{\eta}_{\mathrm{i}}$ are a group of fifteen $4 \times 4$ matrices that, together with the $4 x 4$ identity matrix, compound a basis for all $4 x 4$ matrices. They are also known as Gamma matrices or Dirac Gamma matrices, which arise in quantum electrodynamics. They can be defined from the $2 \times 2$ Pauli matrices $\sigma_{\mathrm{i}}$ and the $2 \times 2$ identity matrix by means of a Kronecker product:

$$
\left.\begin{array}{l}
\boldsymbol{\rho}_{i}=\boldsymbol{\sigma}_{0} \otimes \boldsymbol{\sigma}_{i} \\
\boldsymbol{\rho}_{i}^{\prime}=\boldsymbol{\sigma}_{i} \otimes \boldsymbol{\sigma}_{0}
\end{array}\right\} \Rightarrow \boldsymbol{\eta}_{4 i+j}=\boldsymbol{\rho}_{i}^{\prime} \cdot \boldsymbol{\rho}_{j} .
$$

The use of the Dirac matrices allows the connection of the Mueller matrix elements $m_{i j}$ with a $4 x 4$ coherence matrix, $\mathbf{C}_{\mathbf{4 x} \mathbf{4}}$, which is directly related with the properties of the optical device. This $4 \times 4$ coherence matrix is defined from the $S U(4) \leftrightarrow O^{+}(6)$ homomorphism by the Mueller matrix elements and Dirac matrices as follows [6,7,8]:

$$
\begin{aligned}
& \mathbf{C}_{4 \mathbf{x} 4}=\frac{1}{4} \sum_{i, j=0}^{3} m_{i j} \eta_{i j} \\
& \boldsymbol{\eta}_{0}=\left[\begin{array}{cccc}
1 & 0 & 0 & 0 \\
0 & 1 & 0 & 0 \\
0 & 0 & 1 & 0 \\
0 & 0 & 0 & 1
\end{array}\right] \quad \boldsymbol{\eta}_{1}=\left[\begin{array}{cccc}
0 & 1 & 0 & 0 \\
1 & 0 & 0 & 0 \\
0 & 0 & 0 & i \\
0 & 0 & -i & 0
\end{array}\right] \quad \boldsymbol{\eta}_{2}=\left[\begin{array}{cccc}
0 & 0 & 1 & 0 \\
0 & 0 & 0 & -i \\
1 & 0 & 0 & 0 \\
0 & i & 0 & 0
\end{array}\right] \quad \boldsymbol{\eta}_{3}=\left[\begin{array}{cccc}
0 & 0 & 0 & 1 \\
0 & 0 & i & 0 \\
0 & -i & 0 & 0 \\
1 & 0 & 0 & 0
\end{array}\right] \\
& \boldsymbol{\eta}_{4}=\left[\begin{array}{cccc}
0 & 1 & 0 & 0 \\
1 & 0 & 0 & 0 \\
0 & 0 & 0 & -i \\
0 & 0 & i & 0
\end{array}\right] \quad \boldsymbol{\eta}_{5}=\left[\begin{array}{cccc}
1 & 0 & 0 & 0 \\
0 & 1 & 0 & 0 \\
0 & 0 & -1 & 0 \\
0 & 0 & 0 & -1
\end{array}\right] \quad \boldsymbol{\eta}_{6}=\left[\begin{array}{cccc}
0 & 0 & 0 & -i \\
0 & 0 & 1 & 0 \\
0 & 1 & 0 & 0 \\
i & 0 & 0 & 0
\end{array}\right] \quad \boldsymbol{\eta}_{7}=\left[\begin{array}{cccc}
0 & 0 & 1 & 0 \\
0 & 0 & 0 & -i \\
1 & 0 & 0 & 0 \\
0 & i & 0 & 0
\end{array}\right] \\
& \boldsymbol{\eta}_{\mathbf{8}}=\left[\begin{array}{cccc}
0 & 0 & 1 & 0 \\
0 & 0 & 0 & i \\
1 & 0 & 0 & 0 \\
0 & -i & 0 & 0
\end{array}\right] \quad \boldsymbol{\eta}_{9}=\left[\begin{array}{cccc}
0 & 0 & 0 & i \\
0 & 0 & 1 & 0 \\
0 & 1 & 0 & 0 \\
-i & 0 & 0 & 0
\end{array}\right] \quad \boldsymbol{\eta}_{10}=\left[\begin{array}{cccc}
1 & 0 & 0 & 0 \\
0 & -1 & 0 & 0 \\
0 & 0 & 1 & 0 \\
0 & 0 & 0 & -1
\end{array}\right] \quad \boldsymbol{\eta}_{11}=\left[\begin{array}{cccc}
0 & -i & 0 & 0 \\
i & 0 & 0 & 0 \\
0 & 0 & 0 & 1 \\
0 & 0 & 1 & 0
\end{array}\right] \\
& \boldsymbol{\eta}_{12}=\left[\begin{array}{cccc}
0 & 0 & 0 & 1 \\
0 & 0 & -i & 0 \\
0 & i & 0 & 0 \\
1 & 0 & 0 & 0
\end{array}\right] \quad \boldsymbol{\eta}_{13}=\left[\begin{array}{cccc}
0 & 0 & -i & 0 \\
0 & 0 & 0 & 1 \\
i & 0 & 0 & 0 \\
0 & 1 & 0 & 0
\end{array}\right] \quad \boldsymbol{\eta}_{14}=\left[\begin{array}{cccc}
0 & i & 0 & 0 \\
-i & 0 & 0 & 0 \\
0 & 0 & 0 & 1 \\
0 & 0 & 1 & 0
\end{array}\right] \quad \boldsymbol{\eta}_{15}=\left[\begin{array}{cccc}
1 & 0 & 0 & 0 \\
0 & -1 & 0 & 0 \\
0 & 0 & -1 & 0 \\
0 & 0 & 0 & 1
\end{array}\right]
\end{aligned}
$$

In this case the coherence matrix is describing the polarization properties of the optical medium instead of those of the light. Nevertheless, additional information can be obtained from the eigenvalue analysis of the $4 \times 4$ coherence matrix [9]. A maximum of four non-zero eigenvalues $\lambda_{i}$, with their corresponding eigenvector $\mathbf{C}_{\mathbf{i}}$, can be extracted from its decomposition:

$$
\mathbf{C}_{4 \times 4}=\lambda_{1} \mathbf{C}_{1}+\lambda_{2} \mathbf{C}_{2}+\lambda_{3} \mathbf{C}_{3}+\lambda_{4} \mathbf{C}_{4}
$$


The number of significant (non-zero) eigenvalues and their values are directly related to the depolarizing characteristics of the optical media. In polarization maintaining media, there is only one significant eigenvalue, and then a direct correspondence between the Jones and Mueller matrices can be established [10,11,12]. However, in depolarizing devices, those producing a variation of the polarization degree, there is more than one significant eigenvalue, and the concept of entropy-factor $H$ is then introduced [10,13-15]:

$$
H=-\sum x_{i} \log _{4}\left(x_{i}\right) \rightarrow x_{i}=\frac{\lambda_{i}}{\sum_{j} \lambda_{j}}
$$

If there is only one significant eigenvalue, the entropy-factor takes the value 0 , which corresponds to a perfectly defined output polarization state. In the opposite case, when there are four significant eigenvalues and all of them take the same value, the entropy-factor takes the value 1, which corresponds to a random output polarization state. The entropy-factor will take a value between 0 and $l$, corresponding to all the different situations, according to the number and value of the significant eigenvalues. Therefore, the entropy-factor introduces an independent measurement of the polarization behavior of optical media, which can be properly characterized without any consideration of the input light. According to the values of entropy, three zones can be defined: small value of $\mathrm{H}$ (between 0 and 0.25 ), intermediate value of $\mathrm{H}$ (between 0.25 and 0.8 ), and high value of $\mathrm{H}$ (more than 0.8 ). The depolarization properties of most biological tissues are included into the intermediate region and have a great interest due to the fact that the correlation between the photon pattern and the biological medium is significative.

When the entropy factor does not introduce any significant information, other parameters should be analyzed to obtain an angle-dependent variation of the polarization properties. In this case, the horizontal-to-vertical crosstalk, $H V C$, is calculated. Since in most applications the relative properties of the DUT (Device Under Test) are important, the absolute values of the crosstalk can also lead to valuable information. The horizontal-to-vertical crosstalk ( $H V C$ ) as well as the right-to-left crosstalk $(R L C)$ can be verified [12]. When the incident light is linearly horizontally polarized horizontal-to-vertical crosstalk is defined as the ratio:

$$
H V C=\frac{\text { par }- \text { perp }}{\text { par }+ \text { perp }},
$$

where par corresponds to the intensity of the horizontal polarization output component when irradiating the tissue with horizontally polarized light, and perp is the intensity associated to the vertical component. In order to obtain both intensities, it is not necessary to make the experimental procedure, because from tissue Mueller matrix, Mueller matrices of horizontal and vertical ideal polarizers and the Stokes vector of linearly vertically and horizontally polarized light, they can be calculated by a product of matrices. This parameter $H V C$ shows the isolation degree of the vertical and horizontal components of light coming from the tissue. For instance, if all the incident light is horizontally polarized, then it measures how the tissue depolarises moving the output state of polarization to the vertical axis. More cross-talk parameters can be defined in an analogous way, like VHC (Vertical-to-Horizontal Crosstalk) or LRC (Left-to-Rigth Crosstalk).

All these parameters oscillate between -1 and 1 . In case they present the value 1 , it means that incident light with a concrete polarization state is transformed in an output light with the same state. On the contrary, the value -1 corresponds to a situation in which a concrete incident polarization state would result in a completely opposed output polarization state.

Apart from the process showed before, from the light Stokes vector [16] degrees of total, linear and circular polarization of the output $S_{o}$ can be obtained by means of the following expressions:

$$
S_{o}=\left[\begin{array}{l}
I \\
Q \\
U \\
V
\end{array}\right] \Rightarrow D O P_{\text {total }}=\frac{\sqrt{Q^{2}+U^{2}+V^{2}}}{|I|}
$$




$$
\begin{gathered}
D O P_{\text {linear }}=\frac{\sqrt{Q^{2}+U^{2}}}{|I|} \\
D O P_{\text {circular }}=\frac{\sqrt{V^{2}}}{|I|} .
\end{gathered}
$$

\subsection{Polar decomposition of Mueller matriz}

It is possible to extract precise information about the depolarisation, retardance and diattenuation of a Mueller matrix by means of the so-called polar decomposition proposed by Lu and Chipman [17]. This method decomposes this matrix in a product of three components, $M=M_{\Delta} \cdot \mathrm{M}_{\mathrm{R}} \cdot \mathrm{M}_{\mathrm{D}}$. The decomposition can be also carried out in a reverse form as suggested by R. Ossikovski, A. De Martino and S. Guyot [18], $M=M_{D} \cdot M_{R} \cdot M_{\Delta}$.

The calculation process of these decomposition components will be briefly described now. First of all, the diattenuation component is calculated. From the first row of the Mueller matrix, the diattenuation vector $\vec{D}$ is obtained, and from its unitary vector and module, the submatrix $m_{D}$ is constructed. The diattenuation matrix appears then as:

$$
M_{D}=m_{00}\left[\begin{array}{ll}
1 & \overrightarrow{\mathrm{D}}^{t} \\
\overrightarrow{\mathrm{D}} & \mathrm{m}_{\mathrm{D}}
\end{array}\right] .
$$

The calculus of the depolarisation component $M_{\Delta}$ requires that of the submatrix $\mathrm{m}_{\Delta}$, that is carried out by the following expression:

$$
m_{\Delta}=\varepsilon\left[m^{\prime}\left(m^{\prime}\right)^{\prime}+\left(\sqrt{\lambda_{1} \lambda_{2}}+\sqrt{\lambda_{2} \lambda_{3}}+\sqrt{\lambda_{1} \lambda_{3}}\right) I\right]^{-1} \cdot\left[\left(\sqrt{\lambda_{1}}+\sqrt{\lambda_{2}}+\sqrt{\lambda_{3}}\right) m^{\prime}\left(m^{\prime}\right)^{t}+\sqrt{\lambda_{1} \lambda_{2} \lambda_{3}} I\right] .
$$

With this equation, the tinal expression results:

$$
M_{\Delta}=\left[\begin{array}{ll}
1 & \overrightarrow{0}^{t} \\
\vec{P}_{\Delta} & \mathrm{m}_{\Delta}
\end{array}\right]
$$

The retardance matrix $M_{R}$ can be calculated from the previous ones, and from this matrix several parameters like the total retardance $\mathrm{R}$, the linear retardance $\delta$, the optical rotation $\psi$ and the fast axis orientation respect to the horizontal axis $\theta$ can be obtained [17]. matrix:

An ideal and pure depolarizer, with null retardance and diattenuation, can be expressed by the following Mueller

$$
\left[\begin{array}{llll}
1 & 0 & 0 & 0 \\
0 & \mathrm{a} & 0 & 0 \\
0 & 0 & \mathrm{~b} & 0 \\
0 & 0 & 0 & c
\end{array}\right], \quad|\mathrm{a}|,|\mathrm{b}|,|\mathrm{c}| \leq 1
$$

A natural consequence is that the component $M_{\Delta}$ from the Lu-Chipman decomposition will resemble to this matrix, but it will not be completely identical, due to the fact that in spite of the heterogeneous structure of biological tissues, in general samples measured do not behave like totally depolarising media. 
Due to this similarity, it is convenient to introduce a parameter, called depolarisation power, that comes directly from $\mathrm{a}, \mathrm{b}$ and $\mathrm{c}$ parameters. Like the entropy, it measures the depolarisation introduced by the component $M_{\Delta}$ in a simpler way:

$$
\Delta=1-\frac{|a|+|b|+|c|}{3}, \quad 0 \leq \Delta \leq 1
$$

\section{BACKSCATTERING MUELLER MATRICES ANALYSIS OF BIOLOGICAL TISSUES SUSPENSIONS}

The experimental scheme to obtain the Mueller matrices of the biological tissues in backscattering is shown in Figure 1. The measurement system includes both the input and output polarization devices employed to obtain the Mueller matrix $\mathbf{M}$ of the depolarizing medium. Light coming from an input source is introduced into a polarizer and a quarter-wave plate and its output is sent to a beam splitter $(B S)$. This $B S$ transmits the light on the sample (DUT), focusing it onto the sample through an optical lens. The reflected signal is conducted by the $B S$ to an output analyzer and to the detector.

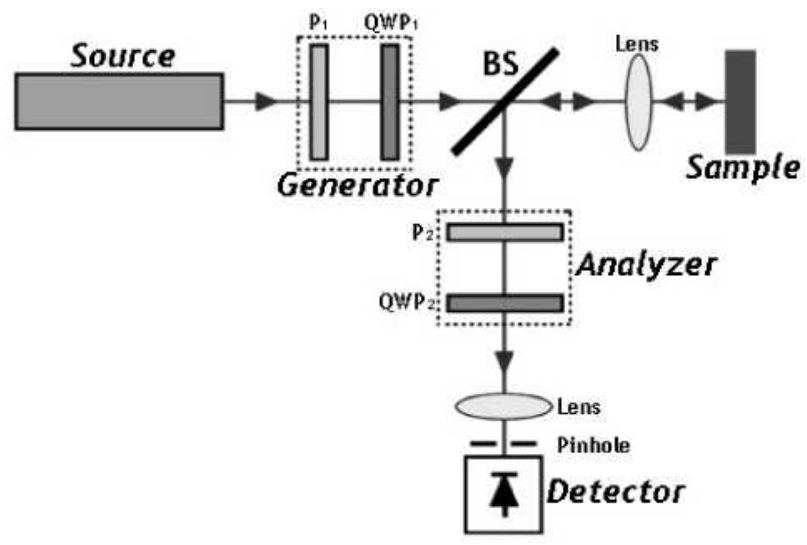

Figure 1. Experimental setup for polarimetry backscattering measurements

Optical devices introduced in the measurement system in order to extract out the Mueller matrix of an optical media can produce depolarization and the introduction of error factors in the measurements because of their imperfections. In this work no depolarization is considered and they are supposed to introduce only first-orders errors, in which no relationship between the orthogonal components or frequency change is produced. These errors are optical activity $\left(\phi_{P}\right)$, strain $\left(\delta_{P}\right)$ and leakage $\left(\gamma_{P}\right)$ in the linear polarizer and optical activity $\left(\phi_{R}\right)$ and dichroism $\left(\alpha_{R}\right)$ in the linear retarder [3]. As polarization devices are supposed to produce no modification of the degree of polarization, they could be analyzed by simple Jones matrix [19]. However, Mueller matrix results are more general, and allow a fast and easy calculation of the whole system to be analyzed, as the DUT's are actual depolarizing media that cannot be analyzed by a Jones matrix. Therefore, the Mueller matrices with the error factors for the linear polarizers and retarders are calculated and the influence of these error-terms on the elements of the Mueller matrix is taken into account.

As a first application example of this procedure, we use a Mueller matrix measured from a glucose solution, whose scattering coefficient is $\mu_{S}=0.6 \mathrm{~mm}^{-1}$ in backscattering [20]. The Mueller matrix of this sample is expressed in the following equation:

$$
M=\left[\begin{array}{cccc}
1 & -0.115 & -0.066 & 0.023 \\
-0.111 & 0.759 & -0.061 & -0.001 \\
-0.018 & 0.151 & -0.435 & -0.139 \\
-0.046 & 0.006 & 0.128 & -0.334
\end{array}\right]
$$


The application of the Lu-Chipman or polar decomposition to this measured matrix gives the following matrices as a result:

$$
\begin{gathered}
M_{D}=\left[\begin{array}{cccc}
1 & -0.115 & -0.066 & 0.023 \\
-0.115 & 1 & 0.0052 & -0.0018 \\
-0.066 & 0.0052 & 0.9939 & -0.0010 \\
0.023 & -0.0018 & -0.0010 & 0.9912
\end{array}\right] \\
M_{\Delta}=\left[\begin{array}{cccc}
1 & 0 & 0 & 0 \\
-0.0282 & 0.7499 & 0.1169 & -0.0060 \\
-0.0266 & 0.1169 & 0.47138 & -0.0093 \\
-0.0297 & -0.0060 & -0.0093 & 0.3589
\end{array}\right] \\
M_{R}=\left[\begin{array}{cccc}
1 & 0 & 0 & 0 \\
-0.0004 & 0.9972 & 0.0599 & 0.0445 \\
0.0001 & 0.0712 & -0.9425 & -0.3264 \\
-0.0001 & 0.0223 & 0.3286 & -0.9441
\end{array}\right] .
\end{gathered}
$$

The Mueller Coherency matrix can also be calculated, as was stated in the previous section, in order to get another parameters set. This Coherency matrix is presented in next equation:

$$
T_{C}=\left[\begin{array}{cccc}
0.495 & -0.113+0.1335 \mathrm{i} & -0.042-0.0035 \mathrm{i} & -0.0115+0.106 \mathrm{i} \\
-0.113-0.1335 \mathrm{i} & 1.264 & 0.045+0.0345 \mathrm{i} & 0.0025+0.024 \mathrm{i} \\
-0.042+0.0035 \mathrm{i} & 0.045-0.0345 \mathrm{i} & 0.07 & -0.0055-0.002 \mathrm{i} \\
-0.0115-0.106 \mathrm{i} & 0.0025-0.024 \mathrm{i} & -0.0055+0.002 \mathrm{i} & 0.171
\end{array}\right]
$$

The method was also applied to a cancerous cell suspension, whose scattering coefficient is $\mu_{\mathrm{S}}=2.2 \mathrm{~cm}^{-1}$. It presents a concentration of $10^{8} \mathrm{cells} / \mathrm{cm}^{3}$. The measurement was made in backscattering with a laser source that presented a wavelength of $\lambda=543 \mathrm{~nm}$. The resulting Mueller matrix is:

$$
M=\left[\begin{array}{cccc}
0.91 & 0.48 & -0.22 & 0.13 \\
0.51 & 0.52 & -0.11 & 0.16 \\
0.05 & -0.01 & -0.108 & -0.07 \\
0.06 & 0.02 & -0.08 & -0.08
\end{array}\right]
$$

In this specific case the Mueller matrix is very near from singularity, what implies some problems for the polar decomposition. After applying it, this three matrices are obtained:

$$
M_{D}=\left[\begin{array}{cccc}
0.91 & 0.48 & -0.22 & 0.13 \\
0.48 & 0.91 & -0.0826 & 0.0488 \\
-0.22 & -0.0826 & 0.7675 & -0.0224 \\
0.13 & 0.0488 & -0.0223 & 0.7428
\end{array}\right]
$$




$$
\begin{aligned}
M_{\Delta} & =\left[\begin{array}{cccc}
1 & 0 & 0 & 0 \\
0.3681 & 0.4023 & -0.0568 & -0.0328 \\
0.0686 & -0.0568 & 0.1384 & 0.0929 \\
0.0738 & -0.0328 & 0.0929 & 0.1155
\end{array}\right] \\
M_{R} & =\left[\begin{array}{cccc}
1 & 0 & 0 & 0 \\
-0.0826 & 0.9591 & -0.1441 & 0.2435 \\
-0.0398 & -0.1443 & -0.9893 & -0.0169 \\
-0.0196 & 0.2433 & -0.0189 & -0.9697
\end{array}\right] .
\end{aligned}
$$

With all these calculated matrices, it is now possible to start the parameters calculation showed in section 2, as a way of making it clearer the polarization information contained in Mueller matrix. Next discussion is devoted to this aim.

\section{DISCUSSION OF RESULTS}

The first case of a glucose suspension will be analysed first. The analysis of the matrices of the polar decomposition makes it possible to obtain the diattenuation $D=0.135$, the total retardance $R=160.867^{\circ}$, the linear retardance $\delta=160.766^{\circ}$, the optical rotation $\psi=5.833^{\circ}$, the fast axis orientation $\theta=13.510^{\circ}$ and the depolarisation power $\Delta=0.473$.

If we take into account the Mueller Coherency matrix, the entropy value can be calculated. In this case, the concrete value is $H_{T}=0.6611$, which is inside the range $0.25 \leq H_{T} \leq 0.8$, previously considered as an optimal study area. This value represents an intermediate-high depolarizing medium [21].

Another parameters that we can calculate are the crosstalks. The horizontal-to-vertical crosstalk results in this case $H V C=0.7322$, and the corresponding vertical-to-horizontal is $V H C=0.78027$. Consequently, these parameters indicate that the medium tends to produce vertically polarized light then it is irradiated with horizontally polarized light in a similar way as vice versa. The tendency is slightly superior in the latter case.

Paying attention to the circular crosstalk values, the right-to-left crosstalk results $R L C=-0.37146$, and the left-toright crosstalk $L R C=-0.29478$. Both of them are negative, and this is consistent with the fact that measurements were made in backscattering and so the mirror effect appears for circularly polarized light. The backscattering process inverts the handedness of circular light.

The same entropy method can be applied to each of the matrices coming from the polar decomposition, instead of doing it with the general Mueller matrix. If this is done with the depolarisation matrix $M_{\Delta}$, the entropy obtained, $H_{T}=0.66923$, is practically coincident with the total entropy calculated before. This is due to the fact that all the depolarization capacity is concentrated in this component.

The result of applying the same analysis to the diattenuation matrix $M_{D}$ gives an almost negligible value of $H_{T}=0.01464$, because this matrix is expected to show only diattenuation and no depolarization. If the crosstalks are extracted, HVC, VHC, RLC and LRC are equal to unity, so the fact of no depolarization is restated.

We also applied the analysis to the retardance matrix $M_{R}$, and similarly it presents a very low entropy value of $H_{T}=0.00168$ like the diattenuation matrix, due to the same fact. The linear crosstalks HVC and VHC are unity, while the circular ones, RLC and LRC, are equal to -1 , what indicates again the handedness inversion for circularly polarized light when measuring in backscattering.

A final analysis is presented in Figure 2, where the eigenvalues of the Mueller Coherency matrix are represented, in order to see their relative importance and influence in the general polarization behaviour of the tissue. 


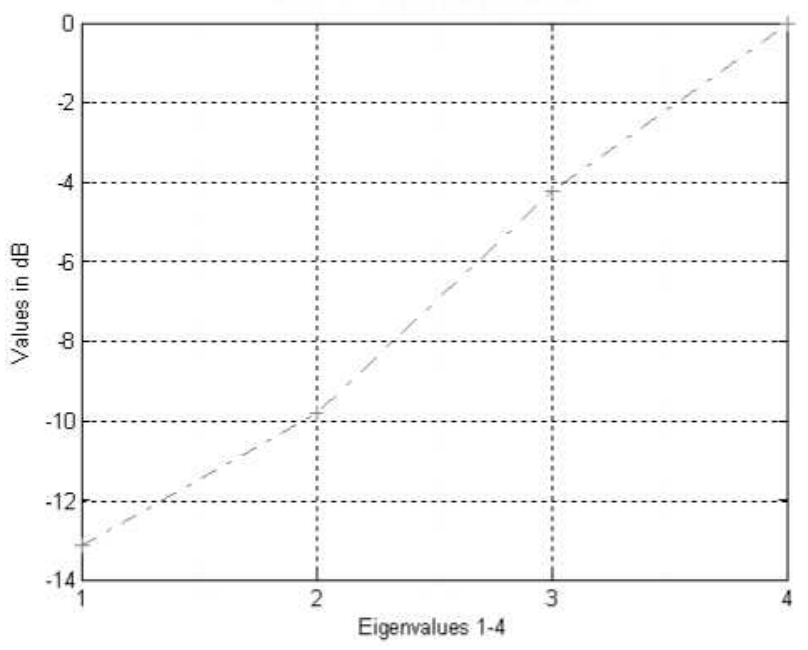

Figure 2. Representation of the Mueller Coherency matriz eigenvalues. In this case, the fourth eigenvalue is bigger than the rest, although it is not dominant due to the fact that the second one is only 2.64 times below $(-4.21 \mathrm{~dB})$.

The analysis is also applied to the cancerous cells suspension, whose polar decomposition was previously shown. In this way, the parameters calculated are the diattenuation $D=0.598$, the total retardance $R=179.944^{\circ}$, the linear retardance $\delta=165.871^{\circ}$, the optical rotation $\psi=89.771^{\circ}$, the fast axis orientation $\theta=-29.675^{\circ}$ and the depolarisation power $\Delta=0.781$.

From the Mueller Coherency matrix, the entropy factor is $H_{T}=0.66278$, which is similar to the value obtained before for the glucose suspension, and indicates that the medium is intermediate-high depolarizing. The linear crosstalks show an interesting behaviour, because $H V C=0.741$ while $V H C=0.0232$, different as in the other suspension, where both were similar. This fact can show that this tissue suspension presents a certain anisotropy in form of characteristic longitudinal structure, what would make the output light to be preferentially oriented in a particular direction. The importance of the orientation of the incident light in the measurement process is seen in this case.

The calculations can go ahead with the circular crosstalks. Here the right-handed circularly polarized light is almost completely depolarized, because $R L C=-0.0192$. This implies that the right-handed light is transformed in the output light in a radiation with almost the same proportion of right and left-handed polarization. For the left-handed circularly polarized light input, the negative value of the crosstalk $L R C=-0.1794$ indicates that the reflected light contains an important amount of right-handed polarized light.

Due to singularity problems with Mueller matrix in this case, no significant results are obtained when applying this method individually to each of the matrices resulting from the polar decomposition, as was perfectly possible in the previous example of a glucose suspension. In this way, the entropy in the depolarization component is not similar to the total entropy of the complete Mueller Coherency matrix, and also the entropy in the other two matrices, diattenuation and retardance, is not near zero.

\section{CONCLUSIONS}

The application of optical polarimetry techniques to characterize biological samples and tissues provides an improved tool in the analysis of tissue properties and structure. The possibility to obtain backscattering Mueller matrices of biological tissues makes possible a non invasive and non contact in-vivo characterization. The introduction of polarization analysis based on Muller Coherency matrix and group theory and /or the Lu-Chipman decomposition makes information appear clearer and contrast increase.

A glucose suspension and a cancerous cells suspension tissue have been analysed using both methods. The corresponding parameters have been calculated and an interpretation has been provided. An exhaustive study of the values of the here proposed polarimetric parameters for different tissues and pathological states should be accomplished in order to provide a clear and confident tool for medical diagnosis in tissue examination, providing an extra instrument 
for the development of optical biopsies. The potentiality of this analysis could be extended to tomographic techniques $[22,23]$, in such a way that not only the parameters coming from one point would be obtained, but the ones corresponding to a complete $3 \mathrm{D}$ biological tissue.

\section{ACKNOWLEDGMENTS}

This work was partially supported by the Spanish Ministry of Education and Science by means of the project TEC2006-06548/TCM.

\section{REFERENCES}

1. T. Vo-Dinh, Biomedical Photonics Handbook, CRC Press, Boca Raton, 2003.

2. V. Sankara, J. T. Walsh and D. J. Maitland, "Comparative study of polarized light propagation in biological tissues", Journal of Biomedical Optics 7(3), 300-306 (2002).

3. D. Pereda Cubián, Č. Vlček, J. L. Arce Diego and Z. Zaoralek, "Variation of the Pauli matrices coefficients in Nddoped fibers subjected to a magnetic field", LEOS'2001, San Diego CA (2001).

4. R. M. A. Azzam and N. M. Bashara, Ellipsometry and Polarized Light, North-Holland, Amsterdam, 1977.

5. H. Mueller, "The foundation of optics", Journal of Optical Society of America A 38, 661 (1948).

6. S. R. Cloude, "Lie groups in electromagnetic wave propagation and scattering", Journal of Electromagnetic Waves and Applications 6(8), 947-974 (1992).

7. J. J. Gil, "Characteristic properties of Mueller matrices", Journal of the Optical Society of America A 17(2), 328$334(2000)$.

8. S. R. Cloude, "Group theory and polarization algebra", Optik 75(1), 26-36 (1986).

9. R. Barakat, "Degree of polarization and the principal idempotents of the coherency matrix", Optics Communications 23(2), 147-150 (1977).

10. D. G. M. Anderson and R. Barakat, "Necessary and sufficient conditions for a Mueller matrix to be derivable from a Jones matrix", Journal of the Optical Society of America A 11(8), 2305-2319 (1994).

11. C. Brosseau, C. R. Givens and A. B. Kostinski, "Generalized trace condition on the Mueller-Jones polarization matrix", Journal of the Optical Society of America A 10(10), 2248-2251 (1993).

12. R. Barakat, "Bilinear constraints between elements of the $4 \times 4$ Mueller-Jones transfer matrix of polarization theory", Optics Communications 38(3), 159-161 (1981).

13. C. Brosseau, "Entropy and the polarization optics: Degree of polarization of a mixture of partially polarized plane waves", Optik 79(3), 117-122 (1988).

14. S. R. Cloude and E. Pottier, "Concept of polarization entropy in optical scattering", Optical Engineering 34(6), 1599-1610 (1995).

15. C. Brosseau, "Polarization transfer and entropy transformation", Optik 88(3), 109-117 (1991).

16. R. Barakat, "Statistics of the Stokes parameters", Journal of the Optical Society of America A 4(7), 1256-1263 (1987).

17. S. Y. Lu and R. A. Chipman, "Interpretation of Mueller matrices based on polar decomposition", Journal of the Optical Society of America A 13(5), 1106-1113 (1996).

18. R. Ossikovski, A. De Martino and S. Guyot, "Forward and reverse product decompositions of depolarizing Mueller matrices", Optics Letters 32(6), 689-691 (2007).

19. J. L. Pezzaniti and R. A. Chipman, "Mueller matrix imaging polarimetry", Optical Engineering 34(6), 1558-1568 (1995).

20. S. Manhas, M. K. Swami, P. Buddhiwant, N. Ghosh, P. K. Gupta and K. Singh, "Mueller matrix approach for determination of optical rotation in chiral turbid media in backscattering geometry", Optical Express 14(1), 190202 (2006).

21. D. Pereda Cubián, J. L. Arce Diego and R Rentmeesters, "Characterization of depolarizing optical media by means of the entropy-factor: Application to biological tissues", Applied Optics 44(3), 358-365 (2005).

22. S. Jiao and L. V. Wang, "Two-dimensional depth-resolved Mueller matrix of biological tissue with double-beam polarization-sensitive optical coherence tomography", Optics Letters 27(2), 101-103 (2002).

23. J. F. de Boer, T. E. Milner, "Review of polarization sensitive optical coherence tomography and Stokes vector determination", Journal of Biomedical Optics 7(3), 359-371 (2002). 